\title{
Prognostic value of programmed death-ligand 1 status in Japanese patients with renal cell carcinoma
}

\author{
Motohide Uemura ${ }^{1}$. Noboru Nakaigawa ${ }^{2}$. Naoto Sassa ${ }^{3,14} \cdot$ Katsunori Tatsugami $^{4,15} \cdot$ Kenichi Harada $^{5}$. \\ Toshinari Yamasaki $^{6} \cdot$ Nobuaki Matsubara $^{7} \cdot$ Takuya Yoshimoto $^{8} \cdot$ Yuki Nakagawa $^{8} \cdot$ Tamaki Fukuyama $^{9}$. \\ Mototsugu Oya $^{10} \cdot$ Nobuo Shinohara $^{11} \cdot$ Hirotsugu Uemura ${ }^{12}$ - Toyonori Tsuzuki ${ }^{13}$
}

Received: 4 February 2021 / Accepted: 12 July 2021 / Published online: 21 July 2021

(c) The Author(s) 2021, corrected publication 2021

\begin{abstract}
Background Programmed death-ligand 1 (PD-L1) positivity is associated with poor prognosis in renal cell carcinoma (RCC). Because the prognostic impact and effect of confounding factors are less known, we investigated the prognostic significance of PD-L1 expression in Japanese patients with recurrent/metastatic RCC who started systemic therapy in 2010-2015.

Methods This multicenter, retrospective study recruited patients from 29 Japanese study sites who had prior systemic therapy for RCC (November 2018 to April 2019) and stored formalin-fixed paraffin-embedded primary lesion samples. The primary outcome was overall survival (OS) by PD-L1 expression. Secondary outcomes included OS in subgroups and duration of first- and second-line therapies by PD-L1 expression. OS distributions were estimated using Kaplan-Meier methodology.

Results PD-L1 expression (on immune cells [IC] $\geq 1 \%$ ) was observed in 315/770 (40.9\%) patients. PD-L1 positivity was more prevalent in patients with poor risk per both Memorial Sloan Kettering Cancer Center [MSKCC] and International Metastatic RCC Database Consortium, and high-risk pathological features (higher clinical stage, nuclear grade and sarcomatoid features). Median OS for PD-L1-positive patients was 30.9 months (95\% CI 25.5-35.7) versus 37.5 months (95\% CI 34.0-42.6) for PD-L1-negative patients (HR 1.04 [90\% CI 0.89-1.22, $p=0.65$ ]; stratified by MSKCC risk and liver metastases). Propensity score weight (PSW)-adjusted OS was similar between PD-L1-positive and -negative patients (median 34.4 versus 31.5 months; estimated PSW-adjusted HR 0.986).

Conclusions This study suggests PD-L1 status was not an independent prognostic factor in recurrent/metastatic RCC during the study period because PD-L1 positivity was associated with poor prognostic factors, especially MSKCC risk status.
\end{abstract}

Keywords Japan $\cdot$ PD-L1 $\cdot$ Prognosis $\cdot$ Renal cell carcinoma $\cdot$ Recurrence

\section{Introduction}

Targeted therapies (e.g., vascular endothelial growth factor receptor [VEGFR] tyrosine kinase inhibitors [TKIs] and mammalian target of rapamycin [mTOR] inhibitors) have been the standard of care for renal cell carcinoma (RCC) since 2008 in Japan [1]. Immunotherapies targeting the programmed death 1/programmed death-ligand 1 (PD-[L]1) pathway and anti-cytotoxic T-lymphocyte-associated antigen 4 (CTLA-4) recently remodeled the metastatic RCC (mRCC) treatment landscape. Checkpoint inhibitors (CPIs) have demonstrated promising efficacies in the first-line (1L)

Hirotsugu Uemura

huemura@med.kindai.ac.jp

Extended author information available on the last page of the article setting in combination with anti-CTLA-4 or VEGFR TKIs [2-4].

PD-L1 is an established biomarker for antitumor T-cell response in other cancers but not in mRCC [5]. Although better outcomes were noted in PD-L1-positive populations, anti-tumor effects were also observed in PD-L1-negative populations compared with controls[2-4]. Thus, routine PD-L1 testing is not standard for treating $\mathrm{mRCC}$ with CPIs. The prognostic role of PD-L1 expression in RCC has been investigated in several studies. High PD-L1 expression (on tumor cells [TC] or tumor-infiltrating immune cells [IC]) correlated with shorter overall survival (OS) and worse prognostic features (high nuclear grade, sarcomatoid differentiation, higher $\mathrm{T}$ stage, and poor International Metastatic RCC Database Consortium [IMDC] 
risk status) [6-9]. Hence, PD-L1 expression was recently recognized as a negative prognostic factor in RCC [10].

Several patient characteristics can affect clinical outcomes in RCC. Two-year survival rates (per IMDC risk classification) were unsatisfactory for patients with poor risk mRCC (7\% versus 75\% and 53\% [favorable and intermediate]) [11]. Poor outcomes have also been reported for patients with mRCC and sarcomatoid components [12]. Tumor immune phenotype is another potential prognostic biomarker; prognosis for clear cell RCC worsens with cluster of differentiation 8-positive (CD8+) T-cell infiltration into tumor tissue and checkpoint molecule expression [13].

PD-L1 expression may not only be a predictive biomarker for CPI treatment, but also a negative prognostic marker in RCC. Thus, clarifying the mechanism behind its potential prognostic significance is important in understanding RCC biology. In this multicenter, retrospective study, we investigated the prognostic significance of PD-L1 expression on OS in a large cohort of Japanese patients treated with systemic therapy for recurrent/metastatic RCC before CPIs became the standard of care for $1 \mathrm{~L}$ treatment. We also explored whether PD-L1 would remain an independent prognostic factor after adjusting for baseline characteristics, which would increase its clinical significance.

\section{Materials and methods}

\section{Patients}

Samples were collected between November 2018 and June 2019 from 29 Japanese study sites that provided guidelinebased, standard-of-care treatment. Patients aged $\geq 20$ years underwent nephrectomy, had stored formalin-fixed paraffinembedded (FFPE) surgical samples of the primary lesion, and started systemic therapy for recurrent/metastatic RCC between January 2010 and December 2015. Since long-term storage of paraffin blocks generally does not significantly impact immunohistochemistry, there was no limitation on the sample collection time of FFPE surgical samples [14]. Exclusion criteria were coexisting malignancies (from nephrectomy to death) or first-line (1L) treatment with anti-CTLA-4/anti-PD-(L)1 for recurrent/metastatic RCC. Patients with tumor tissue samples were recruited and informed consent was obtained. This study is registered under UMIN (UMIN000034131) and was performed after approval by each institutional review board (IRB) of the 29 study sites. Approval from the IRB of MINS, a non-profit organization, was also obtained.

More details on patients can be found in the supplementary methods in the Online Resource.

\section{Study design and outcomes}

This multicenter, retrospective study compared OS by PD-L1 expression status to determine its prognostic effect. The primary outcome was OS, which is defined as the time from initiation of $1 \mathrm{~L}$ therapy to death from any cause, according to PD-L1 status (positive versus negative). Secondary outcomes included OS in subgroups by PD-L1 status, expression level (four IC levels), and duration of $1 \mathrm{~L}$ and second-line (2L) therapy by PD-L1 status.

\section{Pathology and immunohistochemistry}

Representative FFPE samples were selected by pathologists in each institution and evaluated by a central pathologist. Samples had to be of good quality and had to have an adequate number of tumor cells for PD-L1 evaluation. Pathological assessments were conducted to determine the histologic type such as Fuhrman grade (1-4), World Health Organization (WHO)/International Society of Urologic Pathologists (ISUP) grade (1-4), tumor necrosis, lymphovascular infiltration, sarcomatoid component, and growth pattern $[15,16]$.

All samples were sent to the central laboratory (SRL, Inc.) for hematoxylin and eosin staining and immunohistochemistry staining using standardized protocols. PD-L1 expression was evaluated independently by two central pathologists by immunohistochemistry using the VENTANA SP142 assay (Ventana Medical Systems, Inc., 740-4859). Based on IC expression levels, patients were classified as either PD-L1 negative (IC0; IC $<1 \%$ ), or PD-L1 positive (IC1 [IC: $\geq 1 \%$ but $<5 \%$ ], IC2 [IC: $\geq 5 \%$ but $<10 \%$ ] or IC3 [IC $\geq 10 \%$ ]).

Immune phenotype assessment was performed using CD8 immunostaining [17]. Based on three characterizations of T-cell activity from tumor biopsies, patients were categorized into immune-desert (T-cell absence), immuneexcluded (T-cell accumulation but not in tumor core), and immune-inflamed (T-cells infiltration but not functioning) phenotypes [18, 19].

Details on pathology and immunohistochemistry can be found in the Supplementary methods.

\section{Statistical analyses}

The full analysis set used for this study included all enrolled patients, except those with indeterminate PD-L1 expression. Based on previous reports and clinical significance, a median OS of 26 months for the PD-L1-negative group and 20 months for the PD-L1-positive group was assumed, leading to a hypothesized true HR of 1.3 for this study $[4,6]$. When $80 \%$ power is guaranteed at a significance level of $10 \%$, two-sided, the required number 
of events would be 359 . To ensure the required number of events, and accounting for specimens with indeterminate PD-L1 status as well as dropouts due to consent withdrawal, enrollment of approximately 600 patients was planned. nQuery Advisor 7.0 (Statistical Solutions Ltd, Ireland) was used to calculate the number of events.

OS distributions were estimated using the Kaplan-Meier (KM) method, and CI of the median was estimated using the Brookmeyer-Crowley technique [20]. The superiority hypothesis was tested with a two-sided significance level of $10 \%$ using log-rank test, stratified by MSKCC risk criteria (favorable, intermediate, or poor) and liver metastases. These stratification factors were selected based on expected substantial effects on OS and a previous clinical trial, which evaluated PD-L1 status by SP142, considering the non-randomized nature of this study [2]. Stratified HRs and their 90\% CIs were estimated using the stratified Cox regression model (PD-L1 positive: test arm; PD-L1 negative: control arm). Unstratified and subgroup analyses were also performed. In addition, propensity score weight (PSW) analysis was conducted to evaluate the robustness of the primary analysis [21]. A logistic regression model was used to predict the score for each patient, defined as the probability of being PD-L1 positive (explanatory variables in Supplementary Tables S2, S3). After confirming sufficient overlap in propensity score distribution, matching weights method was applied [22].

\section{Results}

\section{Clinicopathological characteristics by PD-L1 status at initial diagnosis and nephrectomy}

Of 777 samples collected, seven samples were not PD-L1-evaluable. Hence, only 770 patients were recruited (PD-L1 positive: 315 ; PD-L1 negative: 455 ). Characteristics between groups were generally balanced for sex, histological type, lymphovascular invasion, and growth pattern (Table 1). Fewer patients in the PD-L1-positive group had undergone radical nephrectomy than in the PD-L1-negative group (38.1\% versus $57.4 \%$ ).

Immune phenotype (CD8-infiltration type) composition differed between PD-L1-positive (excluded: 74.3\%; inflamed: $16.5 \%$; desert: $9.2 \%$ ) and PD-L1-negative groups (excluded: $31.6 \%$; inflamed: $1.5 \%$; desert: $66.8 \%$; Table 1). Furthermore, PD-L1 positivity was numerically higher in patients with higher clinical stage, higher nuclear grade (WHO/ISUP grade and Fuhrman grade), and sarcomatoid disease. The two groups were imbalanced in sample collection year; PD-L1 positivity was lower in older samples.

\section{Clinical characteristics by PD-L1 status at the time of $1 \mathrm{~L}$ therapy}

PD-L1 positivity was similar based on age and metastatic site, including liver metastases (Table 1). A higher proportion of PD-L1-positive than -negative patients had poor prognostic features at $1 \mathrm{~L}$ therapy: poor MSKCC risk, $17.5 \%$ versus $7.3 \%$; poor IMDC risk, $29.2 \%$ versus $14.9 \%$. Fewer PD-L1-positive patients had the following good prognostic features at 1L therapy: favorable MSKCC risk, $11.4 \%$ versus $29.7 \%$; favorable IMDC risk, $10.8 \%$ versus $26.2 \%$.

\section{Systemic therapy for metastatic/recurrent RCC}

Sunitinib (VEGFR TKI) was the most common 1L therapy for PD-L1-positive and -negative groups (51.7 and 53.4\%; Table 2). Second-line therapies were received by $70.8 \%$ of PD-L1-positive and 75.6\% of -negative patients. Axitinib (VEGFR TKI) was the most common 2L therapy (28.3 and $34.3 \%$ ). Third-line therapy was received by $39.0 \%$ of PD-L1-positive and $44.8 \%$ of -negative patients (Table 2). Everolimus (mTOR inhibitor) was the most common thirdline therapy (11.1 and 13.8\%), and nivolumab was the only CPI received (5.1 and 7.0\%). Fourth-line therapies were received by $15.9 \%$ of PD-L1-positive and $23.3 \%$ of -negative patients.

\section{OS by PD-L1 expression (stratified log-rank test)}

Median OS in PD-L1-positive and -negative patients was 30.9 (95\% CI 25.5-35.7) and 37.5 (95\% CI 34.0-42.6) months, respectively (stratified HR $=1.04$ [90\% CI $0.89-1.22, p=0.65]$, unstratified HR 1.21 [90\% CI 1.04-1.40]; Fig. 1). No statistically significant difference in OS distribution was observed; thus, the primary analysis suggested that PD-L1 status alone had no prognostic significance on outcomes in RCC.

\section{OS by PD-L1 expression (PSW-adjusted analysis)}

Sufficient overlap was observed in the distribution of estimated propensity scores. Histograms of pre- and post-PSW-adjusted distributions for PD-L1-positive and -negative groups are shown in Supplementary Fig. S1. No significant difference in PSW-adjusted OS was observed between the two groups, supporting the interpretation of the stratified analysis (Fig. 2). Median PSW-adjusted OS in the PD-L1-positive and -negative groups were 34.4 and 31.5 months, respectively (estimated PSW-adjusted HR 0.99). Additionally, HR stratified by propensity score per 
Table 1 Clinicopathological characteristics at the time of initial diagnosis and nephrectomy and at the time of first-line treatment, by PD-L1 status

\begin{tabular}{|c|c|c|c|}
\hline \multirow[t]{3}{*}{ Category } & \multicolumn{3}{|l|}{ Full analysis set } \\
\hline & PD-L1 positive (IC1/2/3) & PD-L1 negative (IC0) & Total \\
\hline & $n=315$ & $n=455$ & $N=770$ \\
\hline \multicolumn{4}{|c|}{ At initial diagnosis and nephrectomy } \\
\hline Sex (male), $n(\%)$ & $235(74.6)$ & $356(78.2)$ & $591(76.8)$ \\
\hline \multicolumn{4}{|l|}{ Stage at initial diagnosis, $n(\%)$} \\
\hline I & $28(8.9)$ & $84(18.5)$ & $112(14.5)$ \\
\hline II & $17(5.4)$ & $35(7.7)$ & $52(6.8)$ \\
\hline III & $58(18.4)$ & $111(24.4)$ & $169(21.9)$ \\
\hline IV & $207(65.7)$ & $210(46.2)$ & $417(54.2)$ \\
\hline Unknown & $5(1.6)$ & $15(3.3)$ & $20(2.6)$ \\
\hline \multicolumn{4}{|l|}{ Sample collection year, $n(\%)$} \\
\hline Before 2009 & $58(18.4)$ & $135(29.7)$ & $193(25.1)$ \\
\hline 2010-2012 & $133(42.2)$ & $207(45.5)$ & $340(44.2)$ \\
\hline $2013-2015$ & $123(39.0)$ & $112(24.6)$ & $235(30.5)$ \\
\hline After 2016 & $1(0.3)$ & $1(0.2)$ & $2(0.3)$ \\
\hline \multicolumn{4}{|l|}{ Surgery objective, $n(\%)$} \\
\hline Radical nephrectomy & $120(38.1)$ & $261(57.4)$ & $381(49.5)$ \\
\hline Cytoreductive nephrectomy & $195(61.9)$ & $194(42.6)$ & $389(50.5)$ \\
\hline \multicolumn{4}{|c|}{ Metastatic site at initial diagnosis, $n(\%)^{\mathrm{a}}$} \\
\hline Lung & $134(42.5)$ & $121(26.6)$ & $255(33.1)$ \\
\hline Bone & $59(18.7)$ & $58(12.7)$ & $117(15.2)$ \\
\hline Lymph node & $59(18.7)$ & $45(9.9)$ & $104(13.5)$ \\
\hline Liver & $9(2.9)$ & $22(4.8)$ & $31(4.0)$ \\
\hline Adrenal gland & $15(4.8)$ & $19(4.2)$ & $34(4.4)$ \\
\hline Pleura & $12(3.8)$ & $4(0.9)$ & $16(2.1)$ \\
\hline \multicolumn{4}{|c|}{ Pathological feature by central pathology } \\
\hline \multicolumn{4}{|l|}{ Immune phenotype, $n(\%)$} \\
\hline Excluded & $234(74.3)$ & $144(31.6)$ & $378(49.1)$ \\
\hline Inflamed & $52(16.5)$ & $7(1.5)$ & $59(7.7)$ \\
\hline Desert & $29(9.2)$ & $304(66.8)$ & $333(43.2)$ \\
\hline \multicolumn{4}{|c|}{ Histology (central pathology), $n(\%)$} \\
\hline Clear cell RCC & $293(93.0)$ & $403(88.6)$ & $696(90.4)$ \\
\hline Papillary RCC & $7(2.2)$ & $28(6.2)$ & $35(4.5)$ \\
\hline Chromophobe RCC & $1(0.3)$ & $6(1.3)$ & $7(0.9)$ \\
\hline Spindle cell carcinoma & $6(1.9)$ & $1(0.2)$ & $7(0.9)$ \\
\hline Others & $8(2.5)$ & $17(3.7)$ & $25(3.2)$ \\
\hline \multicolumn{4}{|l|}{ Fuhrman grade, $n(\%)$} \\
\hline 1 & $0(0.0)$ & $1(0.2)$ & $1(0.1)$ \\
\hline 2 & $50(15.9)$ & $203(44.6)$ & $253(32.9)$ \\
\hline 3 & $176(55.9)$ & $198(43.5)$ & 374 (48.6) \\
\hline 4 & $88(27.9)$ & $50(11.0)$ & $138(17.9)$ \\
\hline Indeterminable & $1(0.3)$ & $3(0.7)$ & $4(0.5)$ \\
\hline \multicolumn{4}{|l|}{ WHO/ISUP grade, $n(\%)$} \\
\hline 1 & $0(0.0)$ & $1(0.2)$ & $1(0.1)$ \\
\hline 2 & $67(21.3)$ & $233(51.2)$ & $300(39.0)$ \\
\hline 3 & $132(41.9)$ & $152(33.4)$ & $284(36.9)$ \\
\hline 4 & $115(36.5)$ & $66(14.5)$ & $181(23.5)$ \\
\hline Indeterminable & $1(0.3)$ & $3(0.7)$ & $4(0.5)$ \\
\hline \multicolumn{4}{|l|}{ Necrosis, $n(\%)$} \\
\hline Present & $187(59.4)$ & $144(31.6)$ & $331(43.0)$ \\
\hline Absent & $128(40.6)$ & $309(67.9)$ & $437(56.8)$ \\
\hline Indeterminable & $0(0.0)$ & $2(0.4)$ & $2(0.3)$ \\
\hline \multicolumn{4}{|l|}{ Lymphovascular invasion, $n(\%)$} \\
\hline Present & $93(29.5)$ & $96(21.1)$ & $189(24.5)$ \\
\hline Absent & $203(64.4)$ & $336(73.8)$ & $539(70.0)$ \\
\hline
\end{tabular}


Table 1 (continued)

\begin{tabular}{|c|c|c|c|}
\hline \multirow[t]{3}{*}{ Category } & \multicolumn{3}{|l|}{ Full analysis set } \\
\hline & PD-L1 positive (IC1/2/3) & PD-L1 negative (IC0) & Total \\
\hline & $n=315$ & $n=455$ & $N=770$ \\
\hline Indeterminable & $19(6.0)$ & $23(5.1)$ & $42(5.5)$ \\
\hline \multicolumn{4}{|c|}{ Sarcomatoid component, $n(\%)$} \\
\hline Present & $60(19.0)$ & $27(5.9)$ & $87(11.3)$ \\
\hline Absent & $255(81.0)$ & $428(94.1)$ & $683(88.7)$ \\
\hline \multicolumn{4}{|l|}{ Growth pattern, $n(\%)$} \\
\hline Expansive & $107(34.0)$ & $171(37.6)$ & $278(36.1)$ \\
\hline Infiltrative & $85(27.0)$ & $98(21.5)$ & $183(23.8)$ \\
\hline Indeterminable & $123(39.0)$ & $186(40.9)$ & $309(40.1)$ \\
\hline \multicolumn{4}{|l|}{ At $1 \mathrm{~L}$ therapy } \\
\hline \multicolumn{4}{|l|}{ Metastatic site, $n(\%)^{\mathrm{a}}$} \\
\hline Lung & $215(68.3)$ & $286(62.9)$ & $501(65.1)$ \\
\hline Bone & $78(24.8)$ & $120(26.4)$ & $198(25.7)$ \\
\hline Lymph node & $83(26.3)$ & $87(19.1)$ & $170(22.1)$ \\
\hline Liver & $28(8.9)$ & $51(11.2)$ & $79(10.3)$ \\
\hline Adrenal gland & $19(6.0)$ & $27(5.9)$ & $46(6.0)$ \\
\hline Pancreas & $13(4.1)$ & $17(3.7)$ & $30(3.9)$ \\
\hline Brain & $14(4.4)$ & $15(3.3)$ & $29(3.8)$ \\
\hline Kidney (recurrent) & $11(3.5)$ & $11(2.4)$ & $22(2.9)$ \\
\hline Pleura & $19(6.0)$ & $9(2.0)$ & $28(3.6)$ \\
\hline \multicolumn{4}{|c|}{ Age category (years), $n(\%)$} \\
\hline$<40$ & $2(0.6)$ & $14(3.1)$ & $16(2.1)$ \\
\hline $40 \leq 50$ & $23(7.3)$ & $24(5.3)$ & $47(6.1)$ \\
\hline $50 \leq 60$ & $51(16.2)$ & 85 (18.7) & $136(17.7)$ \\
\hline $60 \leq 70$ & $121(38.4)$ & $170(37.4)$ & $291(37.8)$ \\
\hline$\geq 70$ & $118(37.5)$ & $162(35.6)$ & $280(36.4)$ \\
\hline \multicolumn{4}{|l|}{ Liver metastases, $n(\%)$} \\
\hline Present & $28(8.9)$ & $51(11.2)$ & $79(10.3)$ \\
\hline Absent & $286(90.8)$ & $404(88.8)$ & $690(89.6)$ \\
\hline Unknown & $1(0.3)$ & $0(0.0)$ & $1(0.1)$ \\
\hline \multicolumn{4}{|l|}{ MSKCC risk, $n(\%)$} \\
\hline Favorable & $36(11.4)$ & $135(29.7)$ & $171(22.2)$ \\
\hline Intermediate & $224(71.1)$ & $287(63.1)$ & $511(66.4)$ \\
\hline Poor & $55(17.5)$ & $33(7.3)$ & $88(11.4)$ \\
\hline \multicolumn{4}{|l|}{ IMDC risk, $n(\%)$} \\
\hline Favorable & $34(10.8)$ & $119(26.2)$ & $153(19.9)$ \\
\hline Intermediate & $189(60.0)$ & $268(58.9)$ & $457(59.4)$ \\
\hline Poor & $92(29.2)$ & $68(14.9)$ & $160(20.8)$ \\
\hline
\end{tabular}

ICO/1/2/3 PD-L1 expression level on tumor-infiltrating immune cells; IMDC International Metastatic RCC Database Consortium; MSKCC Memorial Sloan Kettering Cancer Center; $P D-L 1$ programmed deathligand 1; RCC renal cell carcinoma; WHO/ISUP World Health Organization/International Society of Urologic Pathologists

${ }^{\mathrm{a}}$ Only sites with prevalence $\geq 3 \%$ are presented

quartile was 1.045. Data pertaining to PSW analysis are shown in Supplementary Tables S4 and S5.

\section{OS by PD-L1 expression in subgroups}

In subgroups based on MSKCC risk at 1L therapy, there was no significant difference in OS distribution based on PD-L1-positive or -negative status for favorable (median 58.6 versus 59.8 months; unstratified HR 0.92 ; $95 \%$ CI 
Table 2 Summary of systemic therapy for metastatic/recurrent $\mathrm{RCC}$

\begin{tabular}{|c|c|c|c|}
\hline \multirow[t]{3}{*}{ Systemic therapy } & \multicolumn{3}{|l|}{ Full analysis set } \\
\hline & $\begin{array}{l}\text { PD-L1 positive } \\
\text { (IC1/2/3) }\end{array}$ & PD-L1 negative (IC0) & Total \\
\hline & $n=315$ & $n=455$ & $N=770$ \\
\hline \multicolumn{4}{|l|}{$1 \mathrm{~L}$} \\
\hline $\begin{array}{l}\text { Patients who received } 1 \mathrm{~L} \text { therapy, } n(\%)^{\mathrm{a}} \\
\text { VEGFR TKI }\end{array}$ & $315(100.0)$ & $455(100.0)$ & $770(100.0)$ \\
\hline Sunitinib & $163(51.7)$ & $243(53.4)$ & $406(52.7)$ \\
\hline Sorafenib & $43(13.7)$ & $48(10.5)$ & $91(11.8)$ \\
\hline Axitinib & $11(3.5)$ & $24(5.3)$ & $35(4.5)$ \\
\hline Pazopanib & $10(3.2)$ & $18(4.0)$ & $28(3.6)$ \\
\hline \multicolumn{4}{|l|}{ mTOR inhibitor } \\
\hline Temsirolimus & $29(9.2)$ & $21(4.6)$ & $50(6.5)$ \\
\hline Interferon-alpha & $46(14.6)$ & $84(18.5)$ & $130(16.9)$ \\
\hline \multicolumn{4}{|c|}{ Reasons for discontinuation of 1L therapy, $n(\%)$} \\
\hline Progressive disease & $191(60.6)$ & $265(58.2)$ & $456(59.2)$ \\
\hline AEs & $84(26.7)$ & $131(28.8)$ & $215(27.9)$ \\
\hline Others & $31(9.8)$ & $37(8.1)$ & $68(8.8)$ \\
\hline Unknown & $3(1.0)$ & $5(1.1)$ & $8(1.0)$ \\
\hline \multicolumn{4}{|l|}{$2 \mathrm{~L}$} \\
\hline $\begin{array}{l}\text { Patients who received 2L therapy, } n(\%)^{\mathrm{a}} \\
\text { VEGFR TKI }\end{array}$ & $223(70.8)$ & $344(75.6)$ & $567(73.6)$ \\
\hline Axitinib & $89(28.3)$ & $156(34.3)$ & $245(31.8)$ \\
\hline Sunitinib & $40(12.7)$ & $49(10.8)$ & $89(11.6)$ \\
\hline Sorafenib & $14(4.4)$ & $37(8.1)$ & $51(6.6)$ \\
\hline \multicolumn{4}{|l|}{ mTOR inhibitor } \\
\hline Everolimus & $48(15.2)$ & $53(11.6)$ & $101(13.1)$ \\
\hline Temsirolimus & $12(3.8)$ & $18(4.0)$ & $30(3.9)$ \\
\hline \multicolumn{4}{|c|}{ Reasons for discontinuation of $2 \mathrm{~L}$ therapy, $n(\%)$} \\
\hline Progressive disease & $124(39.4)$ & $205(45.1)$ & $329(42.7)$ \\
\hline AEs & $67(21.3)$ & $80(17.6)$ & $147(19.1)$ \\
\hline Others & $19(6.0)$ & $28(6.2)$ & $47(6.1)$ \\
\hline Unknown & $3(1.0)$ & $4(0.9)$ & $7(0.9)$ \\
\hline \multicolumn{4}{|l|}{$3 \mathrm{~L}$} \\
\hline $\begin{array}{l}\text { Patients who received } 3 \mathrm{~L} \text { therapy, } n(\%)^{\mathrm{a}} \\
\text { VEGFR TKI }\end{array}$ & $123(39.0)$ & $204(44.8)$ & $327(42.5)$ \\
\hline Axitinib & $27(8.6)$ & $40(8.8)$ & $67(8.7)$ \\
\hline Sunitinib & $12(3.8)$ & $18(4.0)$ & $30(3.9)$ \\
\hline Pazopanib & $10(3.2)$ & $18(4.0)$ & $28(3.6)$ \\
\hline Sorafenib & $10(3.2)$ & $16(3.5)$ & $26(3.4)$ \\
\hline \multicolumn{4}{|l|}{ mTOR inhibitor } \\
\hline Everolimus & $35(11.1)$ & $63(13.8)$ & $98(12.7)$ \\
\hline Temsirolimus & $10(3.2)$ & $10(2.2)$ & $20(2.6)$ \\
\hline \multicolumn{4}{|l|}{ Checkpoint inhibitor } \\
\hline Nivolumab & $16(5.1)$ & $32(7.0)$ & $48(6.2)$ \\
\hline
\end{tabular}

$1 L$ first-line; $2 L$ second-line; $3 L$ third-line; $A E$ adverse event; $I C 0 / 1 / 2 / 3$ PD-L1 expression level on tumorinfiltrating immune cells; $m$ TOR mammalian target of rapamycin; $P D-L 1$ programmed death-ligand 1; TKI tyrosine kinase inhibitor; $V E G F R$ vascular endothelial growth factor receptor

${ }^{a}$ Only therapies with prevalence $\geq 3 \%$ are presented 
Fig. $1 \mathrm{KM}$ curves of OS by PD-L1 status. $C I$ confidence interval; $H R$ hazard ratio; $K M$ Kaplan-Meier; mo months; $O S$ overall survival; $P D-L 1$ programmed death-ligand 1
Fig. 2 KM curves of PSWadjusted OS. $H R$ hazard ratio; $K M$ Kaplan-Meier; mo months; $O S$ overall survival; $P D-L 1$ programmed death-ligand $1 ; P S W$ propensity score-weighted
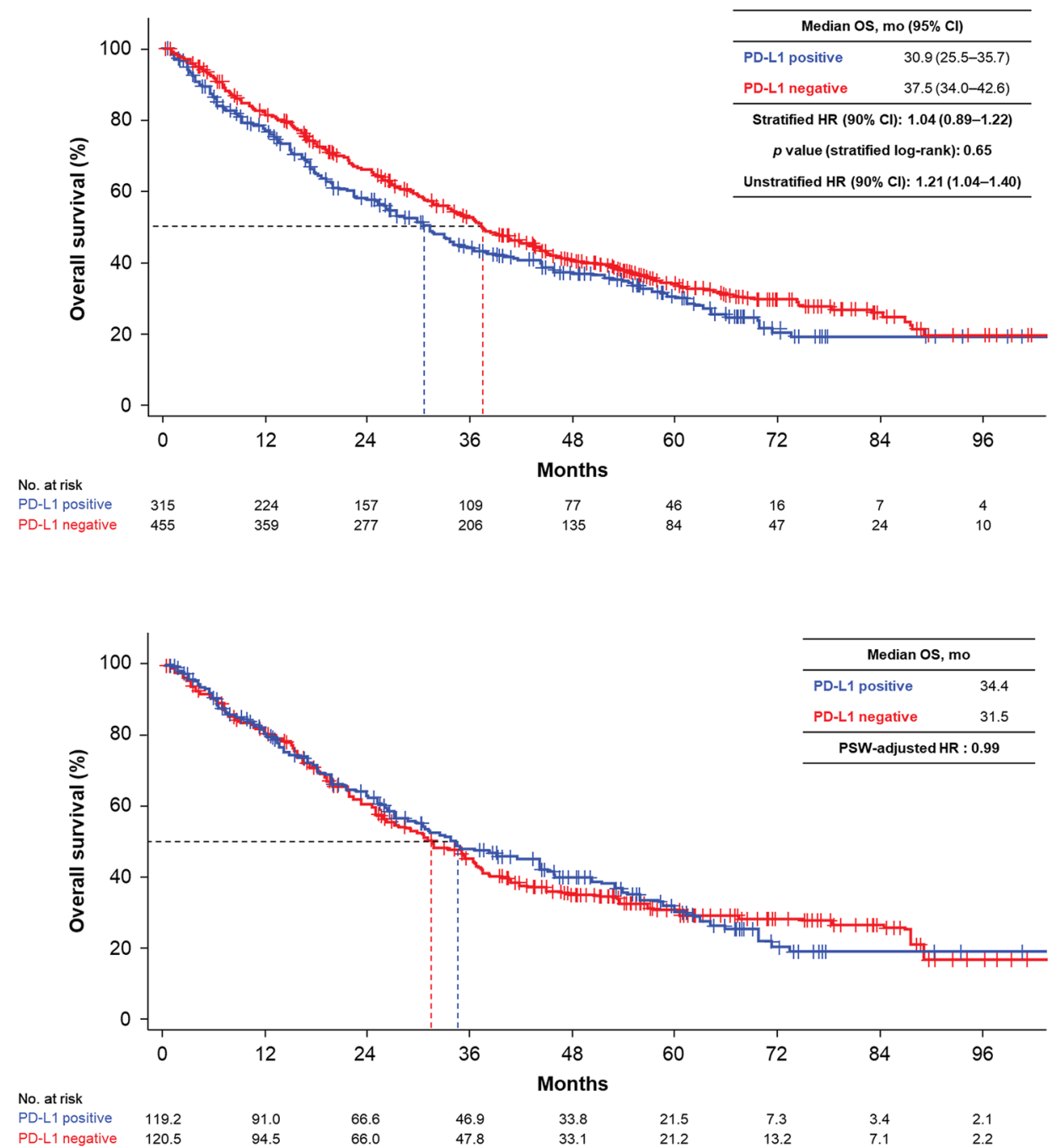

0.54-1.56), intermediate (median 31.6 versus 33.5; HR 1.06; 95\% CI $0.85-1.32$ ) or poor (median 6.6 versus 12.8; unstratified HR 0.89; 95\% CI 0.55-1.43) categories (Figs. 3 and 4). Subgroup analysis of OS by liver metastases status at the time of $1 \mathrm{~L}$ therapy showed a trend towards shorter OS for PD-L1-positive versus PD-L1-negative patients (median OS for absent: 31.6 versus 40.2 months; unstratified HR 1.24; $95 \%$ CI 1.02-1.50, present: 9.0 versus 25.3; unstratified HR 1.27; 95\% CI 0.72-2.23; Fig. 3, Supplementary Fig. S2). Results from other subgroups (based on nuclear grade, IMDC risk criteria, or immune phenotype; Fig. 3, Supplementary Fig. S3-S7) are also reported.

\section{OS by CPI treatment and PD-L1 status}

Of 559 patients who received $\geq 2 \mathrm{~L}$ therapy, 108 received nivolumab after 2L therapy (CPI-treated). Median OS observed for PD-L1-positive versus - negative patients in the CPI-treated group was 71.5 versus 69.4 months (unstratified HR $0.61 ; 95 \%$ CI $0.31-1.21$ ) and 27.2 versus 34.0 months (unstratified HR 1.24; 95\% CI 1.00-1.55) in the CPI-untreated group. In these two groups, KM curves showed a reverse trend (Fig. 4D, E). The analyses in patients who never received CPI throughout their treatment are shown in Supplementary Fig. S8.

\section{Treatment duration in $1 \mathrm{~L}$ and $2 \mathrm{~L}$ settings by PD-L1 status}

Treatment duration in the $1 \mathrm{~L}$ setting was slightly shorter in the PD-L1-positive group than in the PD-L1-negative group (median 5.1 months [95\% CI 4.3-5.9] versus 6.0 months [95\% CI 5.3-7.0]; unstratified HR 1.102 [95\% CI 0.95-1.28]; stratified HR 1.01 [95\% CI 0.87-1.17]; Supplementary Fig. S9a).

Treatment duration of $2 \mathrm{~L}$ therapy was also shorter in the PD-L1-positive group compared with the PD-L1-negative group (median 4.2 months [95\% CI 3.4-5.1] versus 5.7 months [95\% CI 4.9-6.7]; unstratified HR 1.22 [95\% CI 1.03-1.46]; stratified HR 1.17 [95\% CI 0.97-1.40]; Supplementary Fig. S9b). 


\begin{tabular}{|c|c|c|c|c|c|c|c|c|c|c|c|}
\hline \multirow[b]{2}{*}{ Subgroup } & \multirow[b]{2}{*}{ Category } & \multirow[b]{2}{*}{$\mathrm{N}$} & \multicolumn{2}{|c|}{ PD-L1 positive } & \multicolumn{2}{|c|}{ PD-L1 negative } & \multirow[b]{2}{*}{$\mathrm{HR}$} & \multirow[b]{2}{*}{$95 \% \mathrm{Cl}$} & \multirow[b]{3}{*}{0.1} & \multirow[b]{3}{*}{1} & \multirow[b]{3}{*}{10} \\
\hline & & & $\mathbf{n}$ & $\begin{array}{c}\text { Median OS, } \\
\text { mo }\end{array}$ & $\mathbf{n}$ & $\begin{array}{c}\begin{array}{c}\text { Median OS, } \\
\text { mo }\end{array} \\
\end{array}$ & & & & & \\
\hline & & & & & & & & & & & \\
\hline FAS & FAS & 770 & 315 & 30.9 & 455 & 37.5 & 1.206 & {$[1.007-1.445]$} & & & \\
\hline \multicolumn{12}{|c|}{ MSKCC risk criteria } \\
\hline & Favorable & 171 & 36 & 58.6 & 135 & 59.8 & 0.921 & {$[0.543-1.562]$} & & & \\
\hline & Intermediate & 511 & 224 & 31.6 & 287 & 33.5 & 1.059 & [0.851-1.318] & & & \\
\hline & Poor & 88 & 55 & 6.6 & 33 & 12.8 & 0.888 & [0.553-1.425] & & & \\
\hline \multicolumn{12}{|c|}{ IMDC risk criteria } \\
\hline & Favorable & 153 & 34 & 58.6 & 119 & 57.7 & 0.917 & {$[0.530-1.584]$} & & & \\
\hline & Intermediate & 457 & 189 & 33.6 & 268 & 35.1 & 1.043 & [0.823-1.322] & & & \\
\hline & Poor & 160 & 92 & 15.0 & 68 & 19.3 & 1.107 & [0.774-1.583] & & & \\
\hline \multicolumn{12}{|c|}{ Liver metastases at the time of $1 \mathrm{~L}$ therapy } \\
\hline & No & 690 & 286 & 31.6 & 404 & 40.2 & 1.237 & {$[1.021-1.498]$} & & & \\
\hline & Yes & 80 & 29 & 9.0 & 51 & 25.3 & 1.268 & {$[0.722-2.227]$} & & & \\
\hline \multicolumn{12}{|c|}{ Central pathological assessment } \\
\hline \multicolumn{12}{|c|}{ Immune phenotype } \\
\hline & $\begin{array}{l}\text { Excluded } \\
\text { Inflamed }\end{array}$ & $\begin{array}{c}378 \\
59\end{array}$ & $\begin{array}{c}234 \\
52\end{array}$ & $\begin{array}{l}33.6 \\
216\end{array}$ & 144 & $\begin{array}{l}37.8 \\
35.1\end{array}$ & 1.212 & {$[0.929-1.582]$} & & & \\
\hline & Inflamed & 59 & & 21.6 & 7 & 35.1 & 0.978 & {$[0.407-2.348]$} & & & \\
\hline & Desert & 333 & 29 & 19.8 & 304 & 37.1 & 1.529 & {$[0.964-2.424]$} & & & \\
\hline \multicolumn{12}{|c|}{ Fuhrman grade } \\
\hline & Grade1 & 1 & 0 & - & 1 & - & - & - & & & \\
\hline & Grade2 & 253 & 50 & 44.3 & 203 & 55.1 & 1.347 & [0.903-2.008] & & & \\
\hline & Grade3 & 374 & 176 & 34.4 & 198 & 33.5 & 0.947 & {$[0.735-1.221]$} & & & \\
\hline & Grade4 & 138 & 88 & 19.9 & 50 & 17.2 & 0.867 & [0.581-1.293] & & & \\
\hline \multicolumn{12}{|c|}{ WHO/ISUP grade } \\
\hline & Grade1 & 1 & 0 & _- & 1 & _- & _- & _- & & & \\
\hline & Grade2 & 300 & 67 & 50.4 & 233 & 53.7 & 1.035 & [0.715-1.499] & & & \\
\hline & Grade3 & 284 & 132 & 32.5 & 152 & 30.7 & 0.892 & [0.666-1.196] & & & \\
\hline & Grade4 & 181 & 115 & 19.9 & 66 & 21.9 & 1.106 & [0.782-1.565] & & & \\
\hline \multicolumn{12}{|c|}{ Usage of immune check point inhibitors after $2 \mathrm{~L}$ therapy } \\
\hline & No & 650 & 276 & 25.3 & 374 & 31.8 & 1.243 & [1.029-1.502] & & & \\
\hline & Yes & 108 & 35 & 71.5 & 73 & 69.4 & 0.607 & {$[0.306-1.206]$} & & & \\
\hline
\end{tabular}

Fig. 3 Forest plot of OS by PD-L1 status and patient subgroups. $C I$ confidence interval; FAS full analysis set; $H R$ hazard ratio; IMDC International Metastatic RCC Database Consortium; mo months;

\section{Discussion}

Baseline patient characteristics and treatment patterns were similar to those previously reported in a real-world setting in Japan. MSKCC risk status distribution was better than that in the previous report (favorable: $22.2 \%$ versus $13.2 \%$; intermediate: $66.4 \%$ versus $68.1 \%$; poor: $11.4 \%$ versus $18.7 \%$ ) [23].

The proportion of PD-L1-positive patients $(40.9 \%)$ is within the range reported previously $(40-54 \%)[2,24]$. A higher prevalence of PD-L1 positivity was observed in patients with poor prognostic features (i.e., poor MSKCC/ IMDC risk) and high-risk pathological features (higher clinical stage, higher nuclear grade, and sarcomatoid features). These observations are consistent with other reports in patients with high nuclear grade, sarcomatoid component, or high IMDC risk status; patients with clear cell RCC and poor risk status were more likely to express PD-L1 on $\mathrm{TC}$ than those with intermediate or favorable status (poor: 43\%; intermediate: $29 \%$; favorable: $17 \%$; in METEOR study [NCT01865747]) [8, 9, 25]. PD-L1-positive patients
MSKCC Memorial Sloan Kettering Cancer Center; OS overall survival; $P D-L 1$ programmed death-ligand $1 ;$ WHO/ISUP World Health Organization/International Society of Urologic Pathologists

showed more CD8-infiltrated phenotype than PD-L1-negative patients, which aligns with studies describing the correlation of IC status with T-effector gene signature [24].

Median OS after $1 \mathrm{~L}$ treatment was 6 months shorter in the PD-L1-positive than in the -negative group, consistent with previous studies [9]. However, no statistically significant difference in OS distribution was observed between PD-L1-positive and -negative groups when stratified by MSKCC risk and liver metastases. The robustness of this primary analysis was supported by PSW-adjusted analysis. Additionally, in subgroups based on MSKCC risk, OS distributions were very similar for PD-L1-positive and -negative patients and were similarly shortened depending on risk status (Fig. $4 \mathrm{a}-\mathrm{c}$ ). This result aligns with those from the multivariable logistic regression model used to estimate the propensity score, which showed the highest odds ratio with MSKCC risk status, meaning it had the most relevant clinical covariates with PD-L1 expression (Supplementary Tables S2 and S3). MSKCC risk status distribution in PD-L1-positive patients was biased towards a poorer prognosis than in PD-L1-negative patients, suggesting that the OS difference 
Fig. $4 \mathrm{KM}$ curves of OS by PD-L1 status in subgroups after treatment. Subgroups were MSKCC risk criteria A favorable, $\mathbf{B}$ intermediate, and $\mathbf{C}$ poor, and patients who $\mathbf{D}$ used and $\mathbf{E}$ did not use immune checkpoint inhibitor after $2 \mathrm{~L}$ therapy. $2 L$ second-line; $C I$ confidence interval; $H R$ hazard ratio; $K M$ Kaplan-Meier; mo months; $N E$ not evaluable; $O S$ overall survival; $P D-L 1$ programmed death-ligand 1

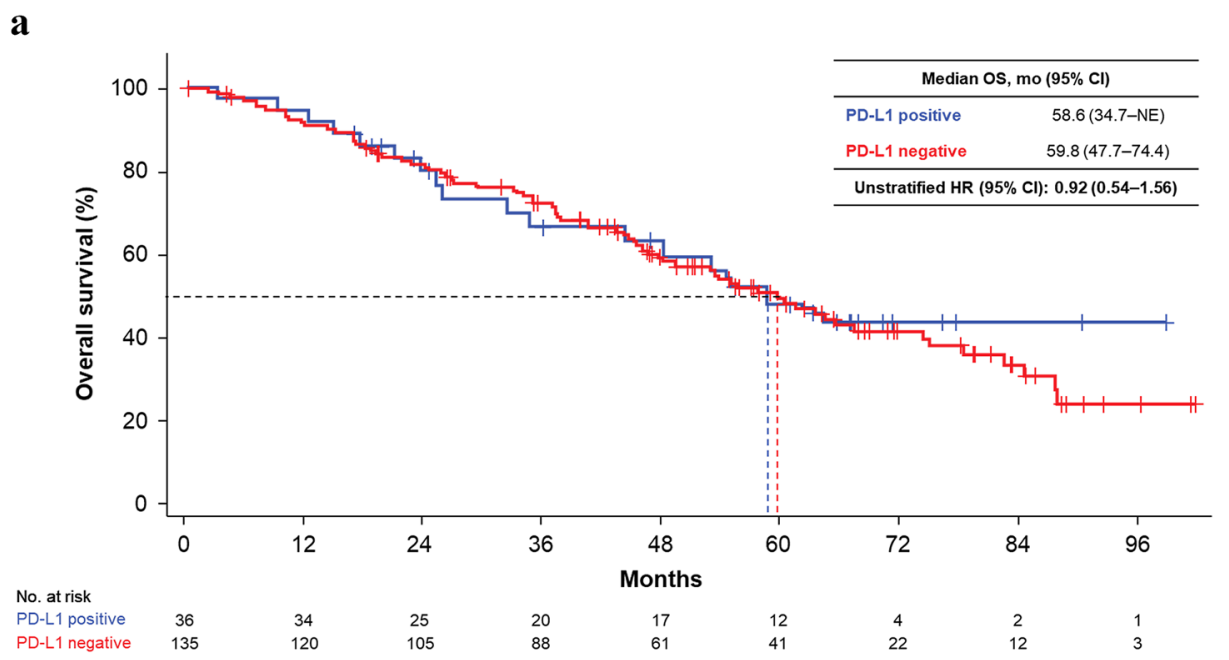

b

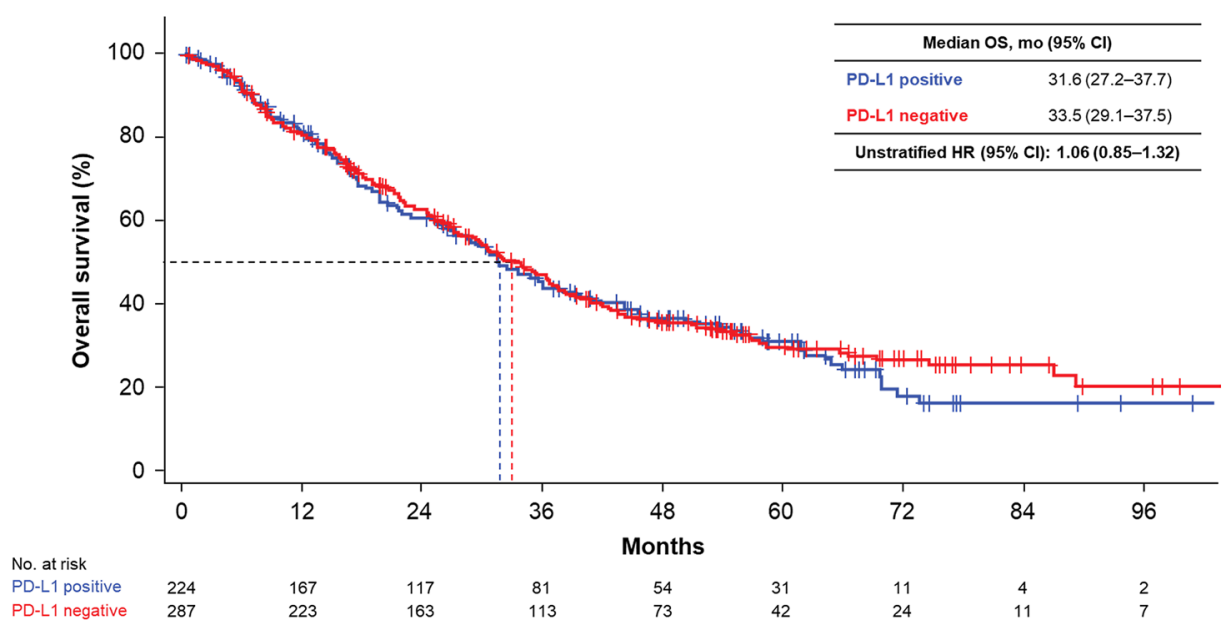

between PD-L1-positive and -negative populations was mainly caused by difference of MSKCC risk status distribution. Therefore, PD-L1 status of primary lesion may not have clinical value when MSKCC/IMDC risk assessment is performed in recurrent/metastatic RCC in the era before CPIs became the standard of care for $1 \mathrm{~L}$ treatment.

The role of PD-L1 expression as a biomarker for CPI response in RCC is not yet established due to its biological complexity (intra/inter patient-heterogeneity and dynamic nature of the marker). Recent studies and a meta-analysis found significantly improved OS and progression-free survival in CPI-treated PD-L1-positive patients [2, 3, 26]. In this study, OS was longer in PD-L1-positive patients who received $\geq 2$ L CPI therapy versus PD-L1-negative patients; the results were reversed in CPI-untreated patients.

New findings in the subgroup analysis by pathological features were observed. Previous studies have shown that low nuclear grades and low CD8 + T-cell infiltration are associated with better outcomes in RCC [13, 27]. This study found a trend towards shorter OS in PD-L1-positive patients with lower Fuhrman grades (e.g., Grade 2; median OS: 44.3 versus 55.1 months; unstratified HR 1.35 [95\% CI 0.90-2.01]) and immune desert phenotype (median OS: 19.8 versus 37.1 months; unstratified HR 1.53 [95\% CI 0.96-2.42]). Interestingly, this observation was not seen in the WHO/ISUP Grade 2 population (Supplementary Fig. S3a and S4a). Nonetheless, this finding suggests that prognosis worsens for patients with RCC if PD-L1-positive ICs infiltrate the tumor, even if other pathological characteristics were favorable. However, among patients with an inflamed phenotype and in subgroups with higher pathological grade, PD-L1-positive patients had similar OS compared with PD-L1-negative patients. These results suggest that a comprehensive examination of PD-L1 (IC) status, malignancy, and CD8 infiltration in surgical specimens may be needed when predicting prognosis.

Due to the retrospective nature of the study, unmeasured confounding factors could have had an effect. This study was 
Fig. 4 (continued)

c

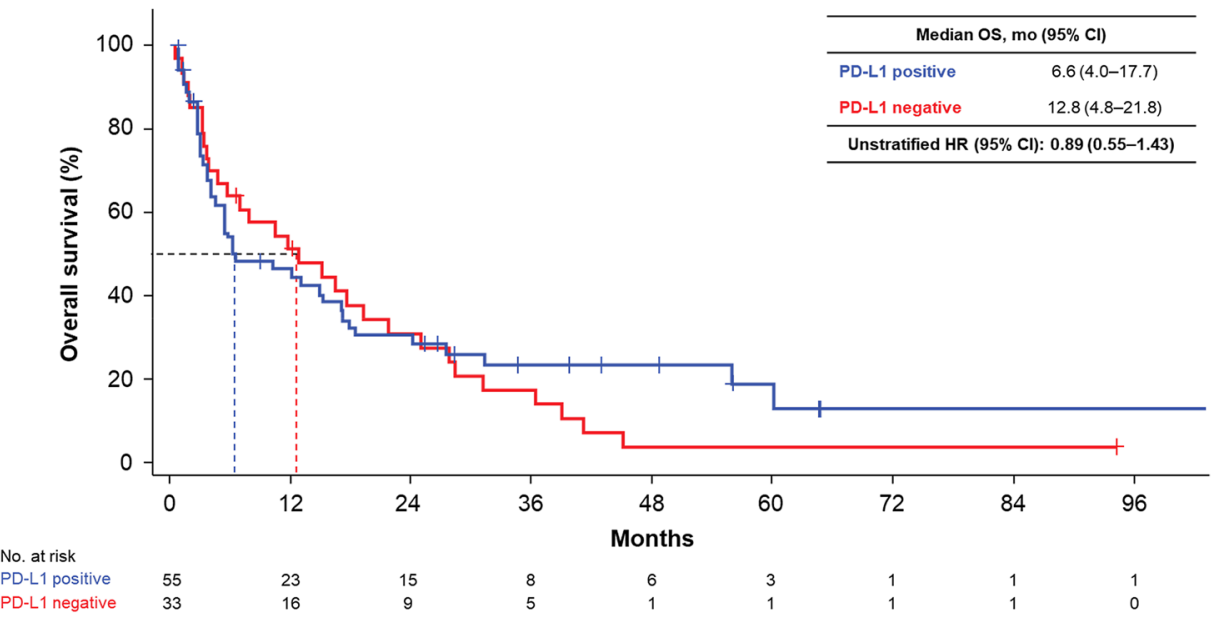

d

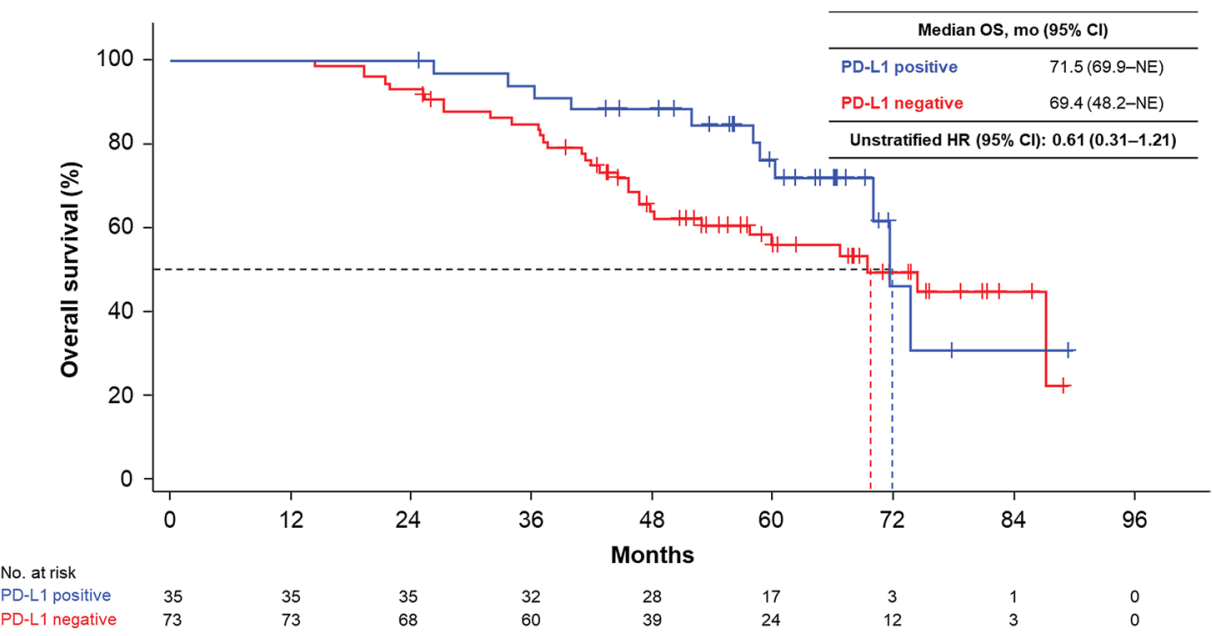

e

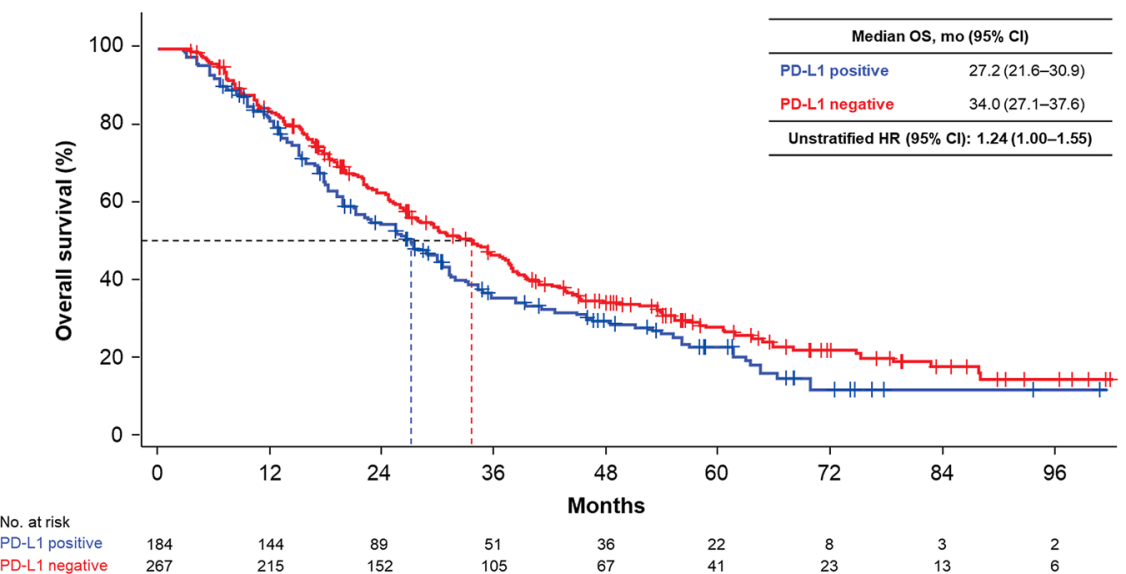

based on $\mathrm{mRCC}$ after nephrectomy, which is representative of patients with RCC in the real world who have undergone nephrectomy but excludes those who have not. Because central pathologists only reviewed selected representative slides, some pathological features could have been underdiagnosed.
Before 2009, the proportion of PD-L1-negative samples was greater than PD-L1-positive ones; however, the trend reversed between 2013 and 2015. A recent study found a strong correlation between high PD-L1 expression and tumor grade in non-small cell lung cancer [28]. In this study, 
PD-L1 positivity was higher in patients with high-risk pathological features (higher clinical stage, higher nuclear grade, and sarcomatoid features; Table 1), and the proportion of patients in Stage I/II was higher during the earlier collection period (Supplementary Table S6). Thus, the difference in PD-L1 positivity across sample collection years was likely to be a result of uneven clinical stage distribution rather than sample storage duration.

These results are clinically relevant in understanding the prognostic value of PD-L1 expression on recurrent/metastatic RCC before CPIs became the standard of care for $1 \mathrm{~L}$ treatment. Overall, this retrospective study was the first to investigate potential associations between clinical outcomes and PD-L1 status in patients with previously treated RCC. Despite the retrospective nature of this study, our results suggest that PD-L1 status is not an independent prognostic factor in recurrent/metastatic RCC because, PD-L1 positivity was associated with other prognostic factors, especially MSKCC risk status.

Supplementary Information The online version contains supplementary material available at https://doi.org/10.1007/s10147-021-01993-x.

Acknowledgements The authors would like to thank the patients who participated in the trial, their families, as well as the investigators and staff at all participating centers (Supplementary Table S1). Ohe Chisato, MD (Department of Pathology and Laboratory Medicine, Kansai Medical University, Japan) contributed to this study by assessing PD-L1 expression of samples. This study was funded by Chugai Pharmaceuticals Co. Ltd. Third-party writing assistance for this manuscript was provided by Bena Lim, PhD, of Health Interactions, and supported by Chugai Pharmaceuticals Co. Ltd.

Author contributions Conceptualization: TT, NM, TF, and HU; Data acquisition: MU, NN, NS, KT, KH, and TY; Data analysis: MO, NS, HU, and TT; Funding acquisition: TF; Statistical analysis: TY, and YN; Writing-draft: MU, NM, TF, and HU; Writing-review and editing: All authors; Supervision: HU.

Funding This study was funded by Chugai Pharmaceuticals Co. Ltd.

\section{Declarations}

Conflict of interest Fukuyama T, Nakagawa Y, and Yoshimoto T are employees of Chugai Pharmaceutical. All honoraria received by the following authors were outside of the submitted work: Uemura $\mathrm{H}$ (Pfizer, MSD, Janssen, BMS, Ono Pharmaceutical, Astellas Pharma, and Bayer), Tsuzuki T (Novartis, Chugai Pharmaceutical, AstraZeneca, Janssen, BMS, Ono Pharmaceutical, Nippon Kayaku, Astellas Pharma, Bayer, Takeda, and Daiichi Sankyo), Oya M (Pfizer, Novartis, AstraZeneca, Janssen, BMS, Ono Pharmaceutical, Astellas Pharma, Bayer, Takeda, and Sanofi), Shinohara N (Pfizer, Novartis, Janssen, BMS, Ono Pharmaceutical, Astellas Pharma, Takeda, and Sanofi).

Open Access This article is licensed under a Creative Commons Attribution 4.0 International License, which permits use, sharing, adaptation, distribution and reproduction in any medium or format, as long as you give appropriate credit to the original author(s) and the source, provide a link to the Creative Commons licence, and indicate if changes were made. The images or other third party material in this article are included in the article's Creative Commons licence, unless indicated otherwise in a credit line to the material. If material is not included in the article's Creative Commons licence and your intended use is not permitted by statutory regulation or exceeds the permitted use, you will need to obtain permission directly from the copyright holder. To view a copy of this licence, visit http://creativecommons.org/licenses/by/4.0/.

\section{References}

1. Yoshimura K, Uemura H (2016) Pharmacotherapies for renal cell carcinoma in Japan. Int J Urol 23(3):194-202. https://doi.org/10. 1111/iju.13008

2. Rini BI, Powles T, Atkins MB et al (2019) Atezolizumab plus bevacizumab versus sunitinib in patients with previously untreated metastatic renal cell carcinoma (IMmotion151): a multicentre, open-label, phase 3, randomised controlled trial. Lancet 393(10189):2404-2415. https://doi.org/10.1016/S0140-6736(19) 30723-8

3. Rini BI, Plimack ER, Stus V et al (2019) Pembrolizumab plus axitinib versus sunitinib for advanced renal-cell carcinoma. N Eng J Med 380(12):1116-1127

4. Motzer RJ, Tannir NM, McDermott DF et al (2018) Nivolumab plus ipilimumab versus sunitinib in advanced renal-cell carcinoma. N Engl J Med 378(14):1277-1290. https://doi.org/10.1056/ NEJMoa1712126

5. Gong J, Chehrazi-Raffle A, Reddi S et al (2018) Development of PD-1 and PD-L1 inhibitors as a form of cancer immunotherapy: a comprehensive review of registration trials and future considerations. J Immunother Cancer 6(1):8-018-0316-z. https://doi.org/10. 1186/s40425-018-0316-z

6. Iacovelli R, Nolè F, Verri E et al (2016) Prognostic role of PD-L1 expression in renal cell carcinoma. A systematic review and metaanalysis. Target Oncol 11(2):143-148. https://doi.org/10.1007/ s11523-015-0392-7

7. Carlsson J, Sundqvist P, Kosuta V et al (2020) PD-L1 expression is associated with poor prognosis in renal cell carcinoma. Appl Immunohistochem Mol Morphol 28:213-220

8. Joseph RW, Millis SZ, Carballido EM et al (2015) PD-1 and PD-L1 expression in renal cell carcinoma with sarcomatoid differentiation. Cancer Immunol Res 3(12):1303-1307. https://doi. org/10.1158/2326-6066.CIR-15-0150

9. Flaifel A, Xie W, Braun DA et al (2019) PD-L1 expression and clinical outcomes to cabozantinib, everolimus, and sunitinib in patients with metastatic renal cell carcinoma: analysis of the randomized clinical trials METEOR and CABOSUN. Clin Cancer Res 25(20):6080-6088. https://doi.org/10.1158/1078-0432. CCR-19-1135

10. Kammerer-Jacquet S-F, Deleuze A, Saout J et al (2019) Targeting the PD-1/PD-L1 pathway in renal cell carcinoma. Int J Mol Sci 20(7):1692

11. Heng DY, Xie W, Regan MM et al (2009) Prognostic factors for overall survival in patients with metastatic renal cell carcinoma treated with vascular endothelial growth factor-targeted agents: results from a large, multicenter study. J Clin Oncol 27(34):57945799. https://doi.org/10.1200/JCO.2008.21.4809

12. Golshayan AR, George S, Heng DY et al (2009) Metastatic sarcomatoid renal cell carcinoma treated with vascular endothelial growth factor-targeted therapy. J Clin Oncol 27(2):235-241. https://doi.org/10.1200/JCO.2008.18.0000

13. Giraldo NA, Becht E, Pagès F et al (2015) Orchestration and prognostic significance of immune checkpoints in the microenvironment of primary and metastatic renal cell cancer. Clin Cancer Res 21(13):3031-3040. https://doi.org/10.1158/1078-0432. CCR-14-2926 
14. Bass BP, Engel KB, Greytak SR et al (2014) A review of preanalytical factors affecting molecular, protein, and morphological analysis of formalin-fixed, paraffin-embedded (FFPE) tissue: how well do you know your FFPE specimen? Arch Pathol Lab Med 138(11):1520-1530. https://doi.org/10.5858/arpa.2013-0691-RA

15. Nishikimi T, Tsuzuki T, Fujita $T$ et al (2011) The post-operative pathological prognostic parameters of clear cell renal cell carcinoma in pT1a cases. Pathol Int 61(3):116-121. https://doi.org/10. 1111/j.1440-1827.2010.02633.x

16. Delahunt B, Eble JN, Egevad L et al (2019) Grading of renal cell carcinoma. Histopathology 74(1):4-17. https://doi.org/10.1111/his.13735

17. Mariathasan S, Turley SJ, Nickles D et al (2018) TGF $\beta$ attenuates tumour response to PD-L1 blockade by contributing to exclusion of T cells. Nature 554(7693):544-548. https://doi.org/10.1038/nature25501

18. Mariathasan S, Turley SJ, Nickles D et al (2017) 8O_PR TGF- $\beta$ signalling attenuates tumour response to PD-L1 checkpoint blockade by contributing to retention of $\mathrm{T}$ cells in the peritumoural stroma. Ann Oncol 28(suppl_11):mdx760.001

19. Binnewies M, Roberts EW, Kersten K et al (2018) Understanding the tumor immune microenvironment (TIME) for effective therapy. Nat med 24(5):541-550. https://doi.org/10.1038/ s41591-018-0014-x

20. Brookmeyer R, Crowley J (1982) A confidence interval for the median survival time. Biometrics 38:29-41

21. Rosenbaum P (1983) The central role of the propensity score in observational studies for causal effects. Biometrika 70:41-55

22. Li L, Greene T (2013) A weighting analogue to pair matching in propensity score analysis. Int J Biostat 9(2):215-234. https://doi. org/10.1515/ijb-2012-0030

23. Harada K, Nozawa M, Uemura M et al (2019) Treatment patterns and outcomes in patients with unresectable or metastatic renal cell carcinoma in Japan. Int J Urol 26(2):202-210. https://doi.org/10. 1111/iju.13830

24. McDermott DF, Huseni MA, Atkins MB et al (2018) Clinical activity and molecular correlates of response to atezolizumab alone or in combination with bevacizumab versus sunitinib in renal cell carcinoma. Nat Med 24(6):749-757. https://doi.org/10. 1038/s41591-018-0053-3

25. Thompson RH, Gillett MD, Cheville JC et al (2004) Costimulatory B7-H1 in renal cell carcinoma patients: indicator of tumor aggressiveness and potential therapeutic target. Proc Natl Acad Sci USA 101(49):17174-17179

26. Roviello G, Corona S, Nesi G et al (2019) Results from a meta-analysis of immune checkpoint inhibitors in first-line renal cancer patients: does PD-L1 matter? Ther Adv Med Oncol 11:1758835919861905. https://doi.org/10.1177/1758835919861905

27. Lang H, Lindner V, de Fromont M et al (2005) Multicenter determination of optimal interobserver agreement using the Fuhrman grading system for renal cell carcinoma: assessment of 241 patients with > 15-year follow-up. Cancer 103(3):625-629. https://doi.org/10.1002/cncr.20812

28. Kerr KM, Thunnissen E, Dafni U et al (2019) A retrospective cohort study of PD-L1 prevalence, molecular associations and clinical outcomes in patients with NSCLC: results from the European Thoracic Oncology Platform (ETOP) Lungscape project. Lung Cancer 131:95-103. https://doi.org/10.1016/j.lungcan.2019. 03.012

Publisher's Note Springer Nature remains neutral with regard to jurisdictional claims in published maps and institutional affiliations.

\section{Authors and Affiliations}

\section{Motohide Uemura $^{1}$ - Noboru Nakaigawa ${ }^{2} \cdot$ Naoto Sassa $^{3,14} \cdot$ Katsunori Tatsugami $^{4,15}$ - Kenichi Harada ${ }^{5}$. Toshinari Yamasaki $^{6} \cdot$ Nobuaki Matsubara $^{7} \cdot$ Takuya Yoshimoto $^{8} \cdot$ Yuki Nakagawa $^{8} \cdot$ Tamaki Fukuyama $^{9}$. Mototsugu Oya $^{10} \cdot$ Nobuo Shinohara $^{11} \cdot$ Hirotsugu Uemura ${ }^{12}$ (1) Toyonori Tsuzuki $^{13}$}

1 Department of Urology, Osaka University Graduate School of Medicine, 2-2 Yamadaoka, Suita, Osaka 565-0871, Japan

2 Department of Urology, Yokohama City University Graduate School of Medicine, 22-2 Seto, Kanazawa Ward, Yokohama, Kanagawa 236-0027, Japan

3 Department of Urology, Nagoya University Graduate School of Medicine, Furocho, Chikusa Ward, Nagoya, Aichi 464-8601, Japan

4 Department of Urology, Kyushu University Graduate School of Medical Sciences, 3-1-1, Maidashi, Higashi-ku, Fukuoka City 812-8582, Japan

5 Division of Urology, Department of Surgery Related, Kobe University Graduate School of Medicine, 7-5-2, Kusunoki-cho, Chuo-ku, Kobe, Hyogo 650-0017, Japan

6 Department of Urology, Kyoto University Graduate School of Medicine, Yoshidakonoecho, Sakyo Ward, Kyoto 606-8501, Japan

7 Department of Breast and Medical Oncology, National Cancer Center Hospital East, 6-5-1 Kashiwanoha, Kashiwa, Chiba 277-8577, Japan

8 Biometrics Department, Chugai Pharmaceutical Co., Ltd., Nihonbashi Muromachi 2-1-1, Chuo City, Tokyo 103-8324, Japan
9 Medical Affairs Division, Chugai Pharmaceutical Co., Ltd., Nihonbashi Muromachi 2-1-1, Chuo City, Tokyo 103-8324, Japan

10 Department of Urology, Keio University School of Medicine, 35 Shinanomachi, Shinjuku City, Tokyo 160-8582, Japan

11 Department of Renal and Genitourinary Surgery, Hokkaido University Graduate School of Medicine, Kita 15, Nishi 7 , Kita-ku, Sapporo 060-8638, Japan

12 Department of Urology, Kindai University, Faculty of Medicine, 377-2 Ohnohigashi, Osaka-Sayama City, Osaka 589-8511, Japan

13 Department of Surgical Pathology, Aichi Medical University Hospital, 1-1 Yazakokarimata, Nagakute, Aichi 480-1195, Japan

14 Present Address: Department of Urology, Aichi Medical University Hospital, 1-1 Yazakokarimata, Nagakute, Aichi 480-1195, Japan

15 Present Address: Department of Urology, Kitakyushu Municipal Medical Center, 2-1-1 Bashaku, Kokurakita Ward, Kitakyushu, Fukuoka 802-0077, Japan 